\title{
Magnetic resonance imaging and ultrasound for assessing parametrial infiltration in cervical cancer. A systematic review and meta-analysis
}

\author{
Juan Luis Alcázar' ${ }^{1}$ Encarnación García ${ }^{2}$, Marcela Machuca $^{3}$, Raquel Quintana ${ }^{4}$, Julia Escrig ${ }^{4}$, \\ Enrique Chacón ${ }^{1}$, José Angel Mínguez ${ }^{1}$, Luis Chiva ${ }^{5}$
}

${ }^{1}$ Department of Obstetrics and Gynecology, Clinica Universidad de Navarra, Pamplona, Spain, ${ }^{2}$ Department of Obstetrics and Gynecology, Virgen de la Arrixaca University Hospital, Murcia, Spain, ${ }^{3}$ Department of Obstetrics and Gynecology, Hospital San José, Santiago, Chile, ${ }^{4}$ Department of Obstetrics and Gynecology, University and Polytechnic Hospital La Fe, Valencia, Spain, ${ }^{5}$ Department of Obstetrics and Gynecology, Clinica Universidad de Navarra, Madrid, Spain

\begin{abstract}
Aims: To provide information on the current evidence regarding the diagnostic performance of ultrasound and MRI for assessing parametrial involvement in cervical cancer using the histological report as the reference standard. Material and methods: Meta-analysis. An extensive search of papers comparing ultrasound and MRI in assessing parametrial infiltration in cervical cancer using pathologic analysis as a reference standard was performed in Medline (Pubmed) and Web of Science from January 1990 to September 2019. Quality was assessed using the QUADAS-2 tool. Results: Our extended search identified 205 citations but after exclusions we finally included 9 articles in the meta-analysis. The risk of bias for most studies was low for four domains were assessed in QUADAS-2. Overall, for ultrasound pooled estimated sensitivity and specificity for diagnosing parametrial infiltration was 78\% (95\% confidence interval $[\mathrm{CI}]: 48 \%-93 \%)$ and 96\% (95\% CI=89\%-99\%), respectively. For MRI these figures were $68 \%(95 \% \mathrm{CI}=54 \%-80 \%)$ and $91 \%(95 \% \mathrm{CI}=84 \%-95 \%)$, respectively. No statistical differences were found when comparing both methods $(\mathrm{p}=0.548)$. Heterogeneity was low/moderate for MRI and high for ultrasound. Conclusion: Ultrasound and MRI have similar diagnostic performance for detecting parametrial infiltration in women with cervical cancer. This might have relevance from the clinical point of view, since ultrasound is cheaper than MRI.

Keywords: uterine cervix; cancer; parametrium; ultrasound; magnetic resonance imaging
\end{abstract}

\section{Introduction}

Cervical cancer is the most frequent gynecological cancer worldwide [1]. Staging of cervical cancer is still

Received 12.12.2019 Accepted 22.01.2020

Med Ultrason

2020, Vol. 22, No 1, 85-91

Corresponding author: Juan Luis Alcazar, MD

Department of Obstetrics and Gynecology,

Clínica Universidad de Navarra

Avenida Pío XII 36, 3110 Pamplona, Spain

$\mathrm{Tel}+34-948-296234$

Fax: +34-948296500

Email: jlalcazar@unav.es based on clinical findings [2]. However, it is well known that discrepancies between clinical examination and the actual extent of disease exist, especially for parametrial involvement [3]. For this reason, imaging methods, namely magnetic resonance (MRI) have been proposed for assessing parametrial infiltration [4].

Traditionally, the role of ultrasound for assessing cervical cancer has been considered very limited [5]. However, some studies have shown the ultrasound (US) might be a good imaging method for assessing the local extension of cervical cancer [6]. In fact, recently a FIGO report has indicated that ultrasound may be a good diagnostic tool for assessing parametrial invasion in cervical can- 
cer [2]. However, to the best of our knowledge no study comparing MRI and ultrasound for assessing parametrial infiltration in a large series, has been reported.

The aim of the present meta-analysis is to provide information on the current evidence regarding the diagnostic performance of ultrasound and MRI for assessing parametrial involvement in cervical cancer using the histological report as the reference standard.

\section{Materials and methods}

\section{Protocol and registration}

We performed this systematic review and meta-analysis according to PRISMA and Synthesizing Evidence from Diagnostic Accuracy TEsts (SEDATE) guidelines [7,8]. All methods for inclusion/exclusion criteria, data extraction and quality assessment were specified in advance. The protocol did not require registration.

\section{Data sources and searches}

Studies published between 1990 and September 2019 were identified by three of the authors (JLA, MM, EG) using two electronic databases (PubMed/MEDLINE and Web of Science) to identify potentially eligible studies. The search terms included and captured the concepts of "cervix," "cancer," "carcinoma," "ultrasound," "sonography," "parametrial," and "magnetic resonance imaging." Language restriction in the research was set to English.

\section{Study selection and data collection}

Three authors (JLA, MM, EG) screened the titles and abstracts identified to exclude irrelevant articles, i.e., those not strictly related to the topic under review. Full-text articles were obtained to identify potentially eligible studies, and reviewers applied independently the following inclusion criteria: 1) prospective or retrospective cohort study including patients who underwent both techniques, MRI and US (those including transvaginal (TVS), transrectal (TRS) or three-dimensional ultrasound), for evaluating parametrial infiltration in cervical carcinoma as index tests. We selected only those studies assessing both techniques in the same set of patients; 2) surgical assessment (radical hysterectomy) of the presence of parametrial infiltration according to the histopathological permanent frozen section as reference standard; 3 ) data reporting of results sufficient to construct the $2 \times 2$ table for diagnostic performance assessment, as a minimum data requirement.

We used the "snowball" strategy to identify potential interesting papers by reading the reference list of those papers selected for full text reading. We attempted to contact the authors in case of missing data.

The Patients, Intervention, Comparator, Outcomes, Study design (PICOS) criteria were used for describing the studies included.
Diagnostic accuracy results and additional useful information about patients and procedures were retrieved from selected primary studies independently by four of the authors (MM, EC, JE, RQ). Disagreements arising during the process of study selection and data collection were resolved by consensus among three of the authors (JLA, JE, RQ).

\section{Risk of bias in individual studies}

Quality assessment was conducted, adapting to this particular review the tool provided by the Quality Assessment of Diagnostic Accuracy Studies-2 (QUADAS-2) [9]. The QUADAS-2 format includes four domains: 1) patient selection, 2) index test, 3 ) reference standard, and 4) flow and timing. For each domain, the risk of bias and concerns about applicability (the latter not applying to the domain of flow and timing) were analyzed and rated as a low, high or unclear risk. The results of quality assessment were used for descriptive purposes to provide an evaluation of the overall quality of the included studies and to investigate potential sources of heterogeneity. Three authors (JLA, JE, RQ) evaluated independently the methodological quality, using a standard form with quality assessment criteria and a flow diagram; they resolved disagreements by discussion between them to reach a consensus.

The methodology of quality criteria was based on the description of inclusion and exclusion criteria for the patient selection domain; a description about how the index test (US/MRI) was performed and interpreted for the index test domain; a description of the reference standard used and a description of the time elapsed from the index test assessment to the reference standard result.

\section{Statistical analysis}

We extracted or derived information on the diagnostic performance of US and MRI. A random-effects model was used to determine overall pooled sensitivity, specificity, positive likelihood ratio (LR+) and negative likelihood ratio (LR-). Positive and negative likelihood ratios (LRs) were used to characterize the clinical utility of the tests and to estimate the post-test probability of disease. A LR of 0.2-5.0 provides weak evidence for either ruling out or confirming the disease. A LR of 5.0-10.0 and $0.1-0.2$ provides moderate evidence to either confirm or rule out the disease. A LR $>10$ or $<0.1$ provides strong evidence to either confirm or rule out the disease.

Using the mean prevalence of parametrial infiltration (pretest probability) in each subset, depending upon the technique assessed and LRs, post-test probabilities were calculated and plotted on Fagan nomograms.

We assessed the presence of heterogeneity for sensitivity and specificity using Cochran's Q statistic and the $\mathrm{I}^{2}$ index [10]. A p-value $<0.1$ indicates heterogeneity. The $\mathrm{I}^{2}$ index describes the percentage of total variation across 
studies that is due to heterogeneity rather than chance. According to Higgins et al. $\mathrm{I}^{2}$ values of $25 \%, 50 \%$, and $75 \%$ would be considered to indicate low, moderate and high heterogeneity, respectively [10]. Forest plots of sensitivity and specificity of all studies were plotted.

Summary receiver-operating characteristics (sROC) curves were plotted to illustrate the relationship between sensitivity and specificity. Comparison of diagnostic performance between US and MRI for detecting parametrial invasion was made using the bivariate method [11]. Meta-regression was used if high heterogeneity was found to assess covariates that could explain this heterogeneity. The covariates analyzed were sample size, prevalence, mean patient age and number of observers (single/multiple) and in the case of US, the technique used (TVS, TRS, 3D). Publication bias was assessed by a regression of diagnostic log odds ratio against $1 / \sqrt{ }$ (effective sample size), weighted by effective sample size, with $\mathrm{p}<0.10$ for the slope coefficient indicating significant asymmetry [12].

All analyses were performed using the Meta-analytical Integration of Diagnostic Accuracy Studies (MIDAS) and METANDI commands in the STATA version 12.0 for Windows (Stata Corporation, College Station, TX, USA). A p-value $<0.05$ was considered as statistically significant.

\section{Results}

\section{Search results}

The electronic search provided a total of 205 citations. After removal of 24 duplicate records, 181 citations remained. Of these, 169 were excluded because it was clear from the title or abstract that they were not relevant to this review (papers assessing US but not MRI [n=2], papers assessing MRI but not US [ $\mathrm{n}=38]$, papers not related to the topic [n=111], reviews [ $\mathrm{n}=18]$ ). We examined the full text of the remaining 12 articles. Finally, six studies were discarded because they did not meet the inclusion criteria (two studies used MRI but not US, three studies

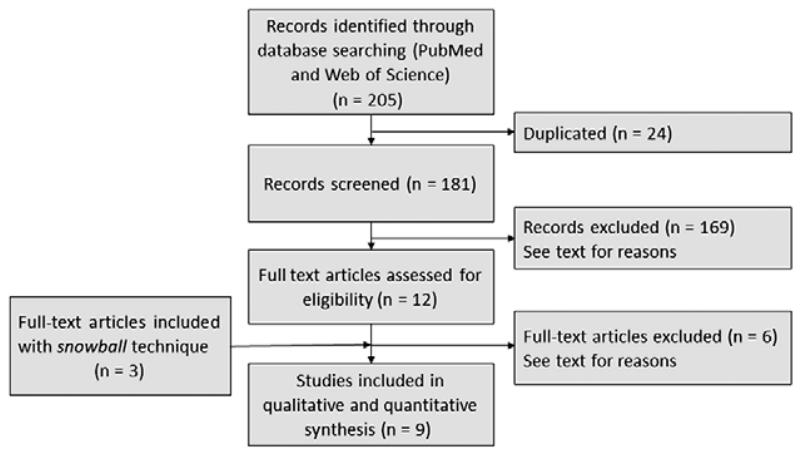

Fig 1. Flow chart showing the studies' selection process had no histopathological analysis as a reference standard and one study used the MRI/US fusion technique).

Thus, the remaining six studies were included in the review and meta-analysis. Three additional relevant studies were found from references cited in the papers included in the review. Thus, a total number of nine studies were included in this meta-analysis [13-21]. A flowchart summarizing literature identification and selection is given in figure 1 .

\section{Characteristics of included studies}

A total of nine studies published between September 1990 and May 2017 reporting on 561 patients were included in the final analyses [13-21]. Among these 561 women, 61 had parametrial invasion. Mean prevalence of parametrial invasion was $14 \%$, ranging from $5 \%$ to $30 \%$. All studies reported the clinical characteristics of the cohort to some extent. Mean patients' age was reported in all studies and ranged from 19 to 84 years. Table I shows PICOS features of the studies included.

\section{Methodological quality of included studies}

Study design was clearly stated as prospective in 7 out of 9 studies [13-18,20]. In two studies, the design was not clearly described $[19,21]$. A graphical display of the evaluation of the risk of bias and concerns regarding applicability of the selected studies is shown in figure. 2 .

Regarding risk of bias and the domain patient selection, one study was not clear regarding the patient inclusion criteria [19] and another study was considered as a high risk for patient selection since it excluded patients with "large cervical cysts, calcifications and necrotic areas, factors which may confound diagnosis results" [21].

Concerning the domain index test, with regard to US, seven studies adequately described the method of the index text as well as how it was performed and interpreted. One study was unclear [14] and one study was considered as a high risk since no description about how US was interpreted was provided [19]. With regard to MRI, four studies adequately described the method of the index text as well as how it was performed and interpreted $[13,15,20,21]$, four studies were unclear [14,16-18] and one study was considered as a high risk since no reporting for MRI evaluation was done [19].

Concerning the domain flow and timing, the time elapsed between the index test and reference standard was reported in all studies apart from one study [20].

For the domain reference standard, all studies were likely to correctly classify the target condition by the reference standard.

Regarding applicability, for the domain patient selection, all studies were deemed to include patients that matched the review question. For the domain index test, four studies were considered as having low concerns 
Table I. Characteristics of included studies in this systematic review according to the PICO criteria

\begin{tabular}{|c|c|c|c|c|c|c|c|c|c|c|c|c|c|}
\hline 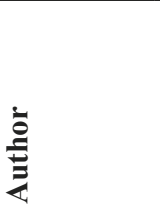 & 离 & 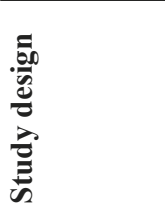 & 离 & Z & 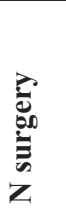 & 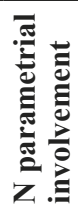 & 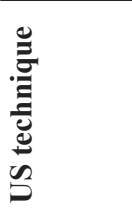 & 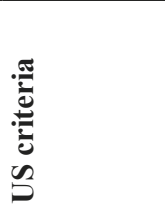 & 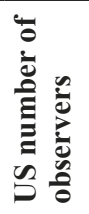 & 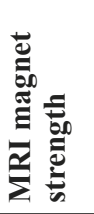 & 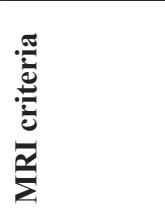 & 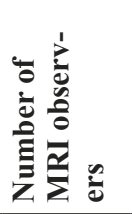 & 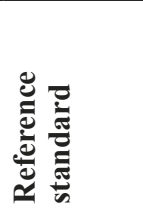 \\
\hline $\begin{array}{l}\text { Cobby } \\
{[13]}\end{array}$ & 1990 & Prospective & No & 37 & 20 & 2 & $\begin{array}{l}\text { 2D TVS/ } \\
\text { TRS }\end{array}$ & $\begin{array}{l}\text { Examiner's } \\
\text { subjective } \\
\text { impression }\end{array}$ & two & $0.5 \mathrm{~T}$ & $\begin{array}{l}\text { Reader's } \\
\text { subjective } \\
\text { impression }\end{array}$ & Two & Histology \\
\hline $\begin{array}{l}\text { Yang } \\
{[14]}\end{array}$ & 1996 & Prospective & No & 38 & 20 & 1 & 2D TRS & $\begin{array}{l}\text { Examiner's } \\
\text { subjective } \\
\text { impression }\end{array}$ & one & $1 \mathrm{~T}$ & $\begin{array}{l}\text { Reader's } \\
\text { subjective } \\
\text { impression }\end{array}$ & Two & Histology \\
\hline $\begin{array}{l}\text { Fischerova } \\
{[15]}\end{array}$ & 2008 & Prospective & No & 95 & 95 & 6 & 2D TRS & $\begin{array}{l}\text { Examiner's } \\
\text { subjective } \\
\text { impression }\end{array}$ & one & $1.5 \mathrm{~T}$ & $\begin{array}{l}\text { Reader's } \\
\text { subjective } \\
\text { impression }\end{array}$ & One & Histology \\
\hline $\begin{array}{l}\text { Testa } \\
{[16]}\end{array}$ & 2009 & Prospective & No & 75 & 68 & 5 & 2D TVS & $\begin{array}{l}\text { Examiner's } \\
\text { subjective } \\
\text { impression }\end{array}$ & one & $1.5 \mathrm{~T}$ & $\begin{array}{l}\text { Reader's } \\
\text { subjective } \\
\text { impression }\end{array}$ & Two & Histology \\
\hline $\begin{array}{l}\text { Epstein } \\
{[17]}\end{array}$ & 2013 & Prospective & Yes & 182 & 182 & 13 & $\begin{array}{l}\text { 2D TVS/ } \\
\text { TRS }\end{array}$ & $\begin{array}{l}\text { Examiner's } \\
\text { subjective } \\
\text { impression }\end{array}$ & $\begin{array}{l}\text { More } \\
\text { than } \\
\text { two }\end{array}$ & $\begin{array}{l}1.5 \mathrm{~T} / \\
3 \mathrm{~T}\end{array}$ & $\begin{array}{l}\text { Reader's } \\
\text { subjective } \\
\text { impression }\end{array}$ & Multiple & Histology \\
\hline $\begin{array}{l}\text { Byun } \\
{[18]}\end{array}$ & 2013 & Prospective & No & 24 & 24 & 4 & 3D TVS & $\begin{array}{l}\text { Examiner's } \\
\text { subjective } \\
\text { impression }\end{array}$ & one & NA & NA & NA & Histology \\
\hline $\begin{array}{l}\text { Han } \\
{[19]}\end{array}$ & 2015 & NA & No & 87 & 80 & 10 & 3D TVS & $\begin{array}{l}\text { Examiner's } \\
\text { subjective } \\
\text { impression }\end{array}$ & one & NA & NA & NA & Histology \\
\hline $\begin{array}{l}\text { Moloney } \\
{[20]}\end{array}$ & 2015 & Prospective & Yes & 46 & 33 & 10 & 2D TVS & $\begin{array}{l}\text { Examiner's } \\
\text { subjective } \\
\text { impression }\end{array}$ & two & $1.5 \mathrm{~T}$ & $\begin{array}{l}\text { Reader's } \\
\text { subjective } \\
\text { impression }\end{array}$ & NA & Histology \\
\hline $\begin{array}{l}\mathrm{Ma} \\
{[21]}\end{array}$ & 2017 & NA & No & 52 & 39 & 11 & 2D TVS & $\begin{array}{l}\text { Examiner's } \\
\text { subjective } \\
\text { impression }\end{array}$ & one & $1.5 \mathrm{~T}$ & $\begin{array}{l}\text { Reader's } \\
\text { subjective } \\
\text { impression }\end{array}$ & one & Histology \\
\hline
\end{tabular}

TVS: transvaginal ultrasound, TRS: transrectal ultrasound, NA: not available, T: tesla. MRI: magnetic resonance imaging, US: ultrasound, $\mathrm{N}$ : number
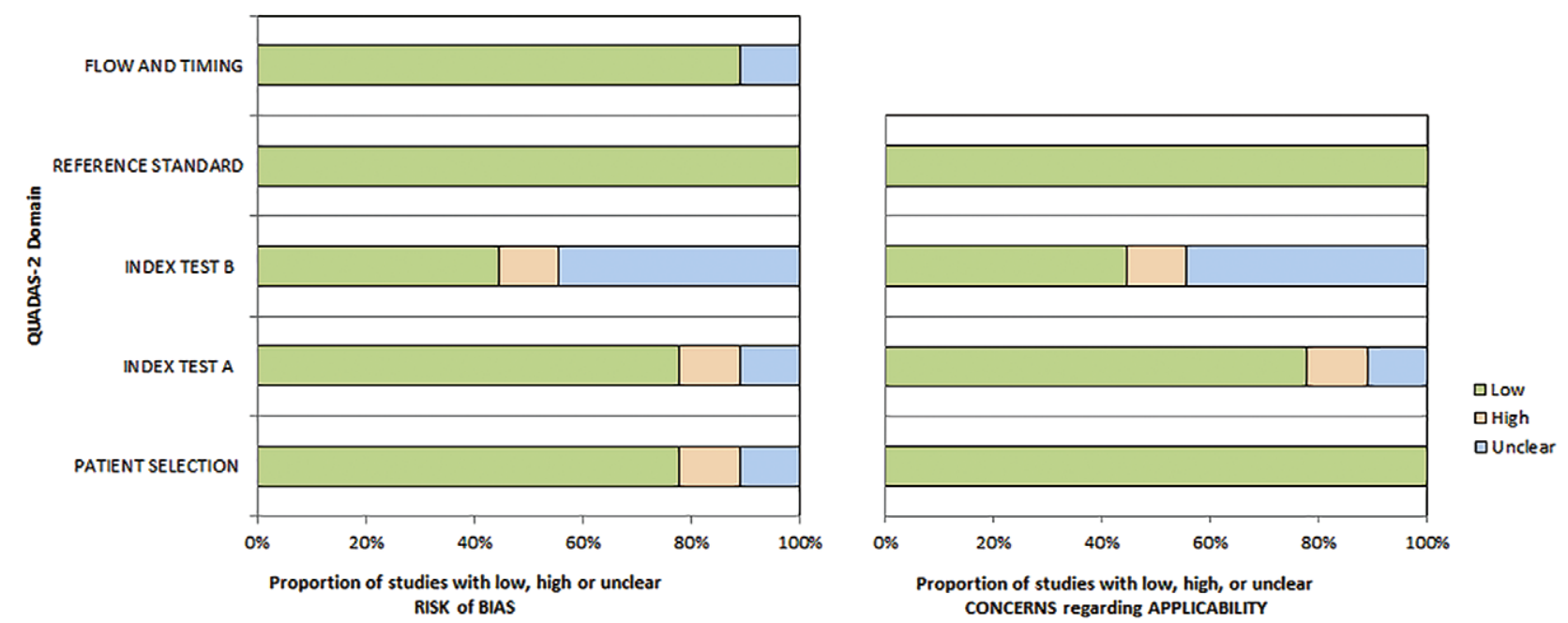

Fig 2. Histogram plot showing the quality assessment (risk of bias and concerns about applicability) for all studies included in the meta-analysis. 


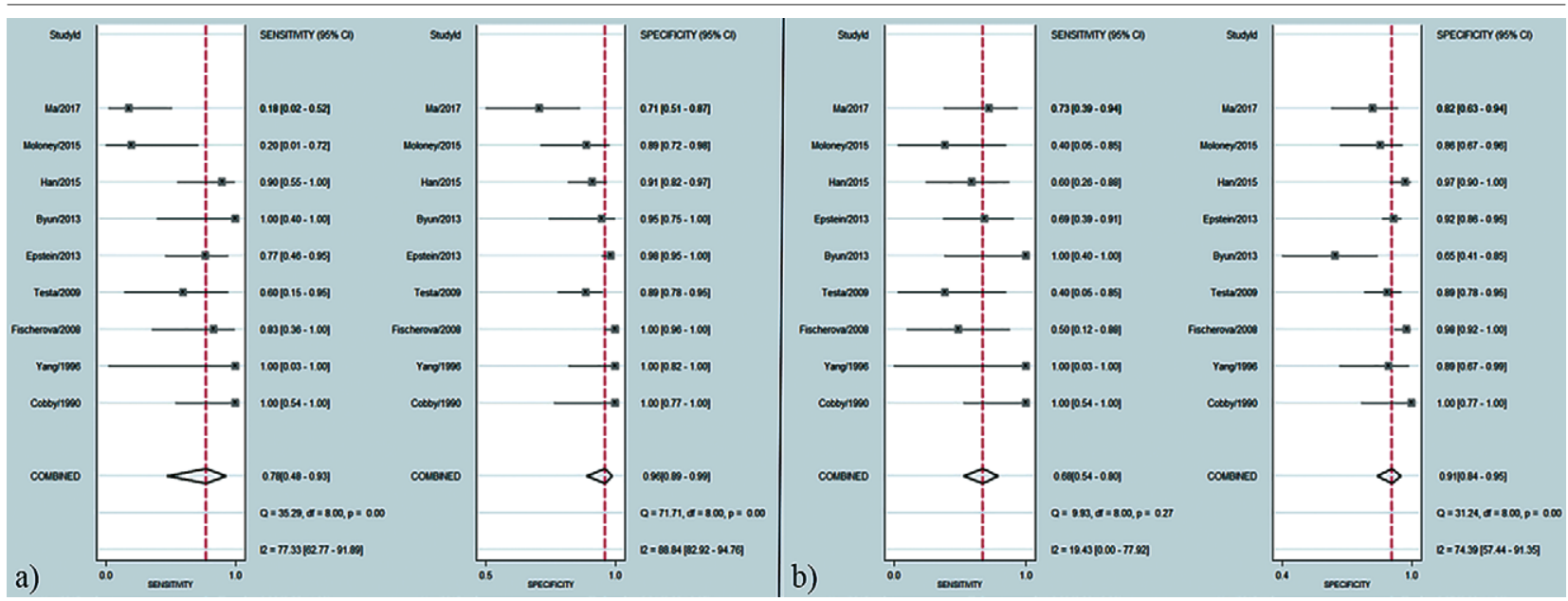

Fig 3. a) Forest plot for sensitivity and specificity for each study and pooled sensitivity and specificity for ultrasound; b) Forest plot for sensitivity and specificity for each study and pooled sensitivity and specificity for MRI

for applicability as the index tests were described well enough for study replication. However, four studies were unclear [16-19] and one was considered as a high risk [14]. All studies presented low concerns regarding the reference standard domain.

\section{Diagnostic performance of TVS and MRI for detection of parametrial infiltration}

Overall, pooled sensitivity, specificity, LR+ and LRof US for detecting parametrial invasion were 78\% (95\% confidence interval $[\mathrm{CI}]=48 \%-93 \%), 96 \%(95 \% \mathrm{CI}=$ $89 \%-99 \%), 20(95 \% \mathrm{CI}=5.5-72)$ and $0.23(95 \% \mathrm{CI}=0.08$ $0.67)$, respectively. High heterogeneity was found for sensitivity $\left(\mathrm{I}^{2}=77.3 \%\right.$; Cochran $\left.\mathrm{Q}=35.29 ; \mathrm{p}<0.01\right)$ and specificity $\left(\mathrm{I}^{2}=88.84 \%\right.$; Cochran $\left.\mathrm{Q}=71.71 ; \mathrm{p}<0.01\right)$.

On the other hand, pooled sensitivity, specificity, LR+, and LR - of MRI for detecting parametrial invasion were 68\% (95\% CI=54\%-80\%), 91\% (95\% CI=84\%-95\%), $7.6(95 \% \mathrm{CI}=4.4-13.2)$, and $0.35(95 \% \mathrm{CI}=0.24-0.52)$, respectively. Low heterogeneity was found for sensitivity ( ${ }^{\mathrm{I}}=19.43 \%$; Cochran $\left.\mathrm{Q}=9.93 ; \mathrm{p}=0.270\right)$ and moderate heterogeneity was found for specificity $\left(\mathrm{I}^{2}=74.39 \%\right.$; Cochran $\mathrm{Q}=31.24$; $\mathrm{p}<0.001$ ).

No statistical differences were found when comparing both methods $(p=0.548)$. Figure 3 shows forest plots for both methods. Meta-regression showed that study design, sample size, prevalence, number of observers (single/ multiple) and type of US examination (2D ultrasound/3D ultrasound) did not explain the heterogeneity observed.

Summary ROC curves are shown in figure 4. Fagan nomograms show that a positive test for US and MRI increases significantly the pretest probability for parametrial infiltration, from $14 \%$ to $76 \%$ in the case of US and from $14 \%$ to $55 \%$ in the case of MRI. While a negative test significantly decreases the pretest probability, from
$14 \%$ to $4 \%$ in the case of US and from $14 \%$ to $5 \%$ in the case of MRI (fig 5).
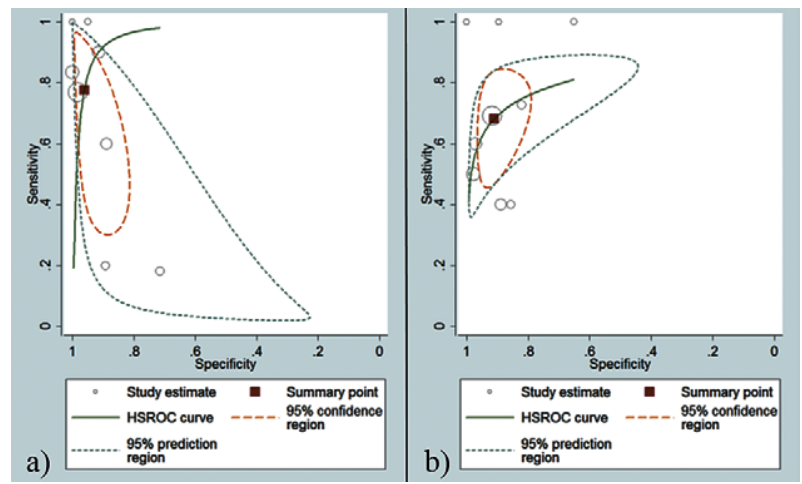

Fig 4. a) Summary ROC curve for ultrasound; b) Summary ROC curve for MRI
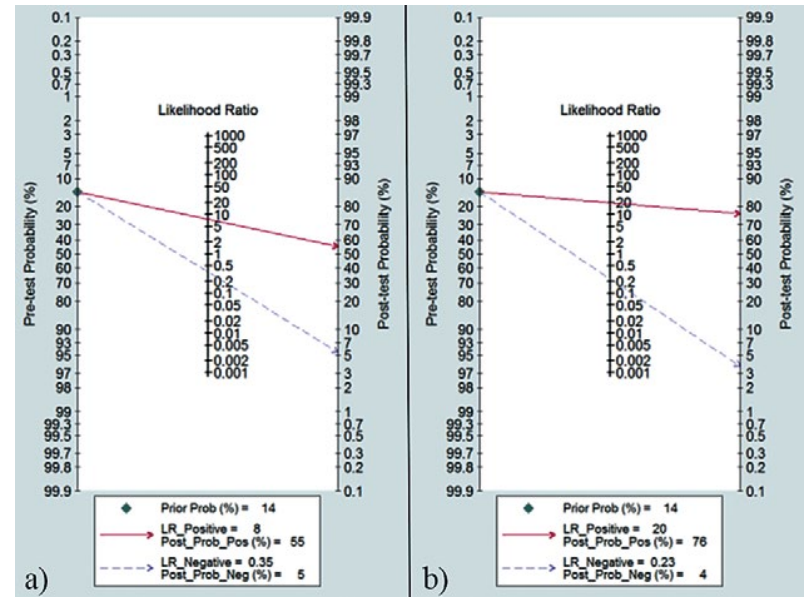

Fig 5. a). Fagan's nomogram for ultrasound; b) Fagan's nomogram for MRI 
No publication bias was found, neither for US $(\mathrm{p}=0.860)$ nor for MRI $(\mathrm{p}=0.760)$.

\section{Discussion}

In the present meta-analysis, we have assessed and compared the diagnostic accuracy of ultrasound and MRI for detecting parametrial infiltration in cervical cancer, using pathological findings as a reference standard. We have found that both techniques have similar accuracy.

The main strength of this study is that, to the best of our knowledge, this is the first meta-analysis that address this issue, comparing both techniques. Another strength of our study is that we only included studies using both techniques in the same set of patients. This allow a direct comparison, reducing the risk of selection bias that could exist if we had compared studies using MRI but not ultrasound with studies using ultrasound but not MRI.

As stated above, there is no meta-analysis reported comparing ultrasound and MRI for assessing parametrial invasion in cervical cancer. However, there are some published meta-analysis assessing MRI accuracy. Bipat et al reported a meta-analysis in 2003 [22]. They included fifty-seven studies using MRI, reported between 1985 and 2002. The reference standard was the histopathology. Pooled sensitivity was $74 \%$ and specificity was about $85 \%$. Heterogeneity among studies was found. Factors such as year of publication, sample size and magnet strength did not explain this heterogeneity. Thomeer et al included 40 studies reported between 1986 and 2011 using MRI in their meta-analysis [23]. However, they included patients with no pathological reference standard. They found that pooled sensitivity and specificity of MRI was $84 \%$ and $92 \%$, respectively. Moderate heterogeneity among studies was observed. Meta-regression was not specifically stated as performed in this study. However, the authors reported that prevalence of parametrial invasion and magnet strength affected the pooled sensitivity and specificity. More recently, Woo et al reported a third meta-analysis using MRI, including 14 studies reported between 2012 and 2016 [24]. The pooled sensitivity and specificity reported were $73 \%$ and $93 \%$, respectively. They also found moderate heterogeneity among studies. Meta-regression showed that magnet strength, use of Diffusion Weighted Imaging (DWI) and anti-spasmodic drugs explained this heterogeneity.

The results of all these three previous meta-analyses, in terms of pooled sensitivity and specificity, are similar to ours. We observed that pooled sensitivity and specificity was $68 \%$ and $91 \%$, respectively. Probably, we observed a rather lower sensitivity because of our selection criteria for studies to be included in the meta-analysis, excluding those studies not assessing ultrasound in the same study.

All three previous meta-analysis and ours, observed significant heterogeneity among studies using MRI. We also found heterogeneity among those studies using ultrasound. Apparently, one factor that could explain this heterogeneity for MRI studies could be magnet strength (observed in two meta-analyses). This heterogeneity among studies can be considered as a limitation for drawing meaningful conclusions regarding the use of MRI in these clinical setting and clearly shows that there is room for future research. We think the same is for ultrasound studies.

Another limitation of our study is the small number of patients included. Therefore, our findings must be interpreted with caution.

Our findings may have clinical relevance, especially when FIGO has stated recently that both techniques can be used for imaging staging of cervical cancer [7]. Ultrasound is much cheaper and more widely available than MRI. This is important, especially when considering that cervical cancer is much more prevalent in less developed countries [1]. Certainly, ultrasound examination should be performed by an experienced examiner. Therefore, training and experience is an important issue. However, there is no published information about the learning curve for ultrasound assessment of cervical cancer. MRI performance might also depend on the experience of the radiologist reading MRI images. Nevertheless, some technical factors may also be relevant, such as the magnet strength and use of DWI.

In conclusion, ultrasound and MRI have a similar diagnostic performance for assessing parametrial infiltration in cervical cancer. However, due to the heterogeneity found among the studies included in this meta-analysis and the relatively small sample size, these findings must be taken with caution. Further well-designed studies would be needed for comparing MRI and ultrasound in the assessment of parametrial invasion in cancer of the uterine cervix.

\section{Conflict of interest: none}

\section{References}

1. Ferlay J, Soerjomataram I, Dikshit R, et al. Cancer incidence and mortality worldwide: sources, methods and major patterns in GLOBOCAN 2012. Int J Cancer 2015;136:E359E386.

2. Bhatla N, Aoki D, Sharma DN, Sankaranarayanan R. Cancer of the cervix uteri. Int J Gynaecol Obstet 2018;143 Suppl 2:22-36.

3. Delgado G, Bundy B, Zaino R, Sevin BU, Creasman WT, Major F. Prospective surgical-pathological study of dis- 
ease-free interval in patients with stage IB squamous cell carcinoma of the cervix: a Gynecologic Oncology Group study. Gynecol Oncol 1990;38:352-357.

4. Lai CH, Yen TC, Ng KK. Surgical and radiologic staging of cervical cancer. Curr Opin Obstet Gynecol 2010;22:15-20.

5. Follen $\mathrm{M}$, Levenback $\mathrm{CF}$, Iyer $\mathrm{RB}$, et al. Imaging in cervical cancer. Cancer 2003;98(9 Suppl):2028-2038.

6. Gaurilcikas A, Vaitkiene D, Cizauskas A, et al. Early-stage cervical cancer: agreement between ultrasound and histopathological findings with regard to tumor size and extent of local disease. Ultrasound Obstet Gynecol 2011;38:707715 .

7. Moher D, Liberati A, Tetzlaff J, Altman DG; PRISMA Group. Preferred reporting items for systematic reviews and meta-analyses: the PRISMA statement. BMJ 2009;339:b2535.

8. Sotiriadis A, Papatheodorou SI, Martins WP. Synthesizing Evidence from Diagnostic Accuracy TEsts: the SEDATE guideline. Ultrasound Obstet Gynecol 2016;47:386395.

9. Whiting PF, Rutjes AW, Westwood ME, et al. QUADAS-2: a revised tool for the quality assessment of diagnostic accuracy studies. Ann Intern Med 2011;155:529- 536.

10. Higgins JP, Thompson SG, Deeks JJ, Altman DG. Measuring inconsistency in meta-analyses. BMJ 2003;327:557560 .

11. EUnetHTA Guideline. Meta-analysis of diagnostic test accuracy studies. Accessed February 2017. Available at: http://www.eunethta.eu/eunethta-guidelines.

12. Deeks JJ, Macaskill P, Irwig L. The performance of tests of publication bias and other sample size effects in systematic reviews of diagnostic test accuracy was assessed. J Clin Epidemiol 2005;58:882-893.

13. Cobby M, Browning J, Jones A, Whipp E, Goddard P. Magnetic resonance imaging, computed tomography and endosonography in the local staging of carcinoma of the cervix. Br J Radiol 1990;63:673-679.

14. Yang WT, Walkden SB, Ho S, et al. Transrectal ultrasound in the evaluation of cervical carcinoma and comparison with spiral computed tomography and magnetic resonance imaging. Br J Radiol 1996;69:610-616.
15. Fischerova D, Cibula D, Stenhova H, et al. Transrectal ultrasound and magnetic resonance imaging in staging of early cervical cancer. Int J Gynecol Cancer 2008;18:766-772.

16. Testa AC, Ludovisi M, Manfredi R, et al. Transvaginal ultrasonography and magnetic resonance imaging for assessment of presence, size and extent of invasive cervical cancer. Ultrasound Obstet Gynecol 2009;34:335-344.

17. Epstein E, Testa A, Gaurilcikas A, et al. Early-stage cervical cancer: tumor delineation by magnetic resonance imaging and ultrasound - a European multicenter trial. Gynecol Oncol 2013;128:449-453.

18. Byun JM, Kim YN, Jeong DH, Kim KT, Sung MS, Lee KB. Three-dimensional transvaginal ultrasonography for locally advanced cervical cancer. Int J Gynecol Cancer 2013;23:1459-1464.

19. Han XS, Ning CP, Sun LT, Li XY, Peng YQ, Dang MZ. Three-dimensional transvaginal tomographic ultrasound imaging for cervical cancer staging. Ultrasound Med Biol 2015;41:2303-2309.

20. Moloney F, Ryan D, Twomey M, Hewitt M, Barry J. Comparison of MRI and high-resolution transvaginal sonography for the local staging of cervical cancer. J Clin Ultrasound 2016;44:78-84.

21. Ma X, Li Q, Wang JL, et al. Comparison of elastography based on transvaginal ultrasound and MRI in assessing parametrial invasion of cervical cancer. Clin Hemorheol Microcirc 2017;66:27-35.

22. Bipat S, Glas AS, van der Velden J, Zwinderman AH, Bossuyt PM, Stoker J. Computed tomography and magnetic resonance imaging in staging of uterine cervical carcinoma: a systematic review. Gynecol Oncol 2003;91:59-66.

23. Thomeer MG, Gerestein C, Spronk S, van Doorn HC, van der Ham E, Hunink MG. Clinical examination versus magnetic resonance imaging in the pretreatment staging of cervical carcinoma: systematic review and meta-analysis. Eur Radiol 2013;23:2005-2018.

24. Woo S, Suh CH, Kim SY, Cho JY, Kim SH. Magnetic resonance imaging for detection of parametrial invasion in cervical cancer: An updated systematic review and metaanalysis of the literature between 2012 and 2016. Eur Radiol 2018;28:530-541. 